\title{
ESTUDO DO COMPORTAMENTO DE FASES DE SISTEMAS REPRESENTATIVOS DE PETRÓLEO COM CO $\mathrm{CO}_{2}$.
}

\author{
I.C.C. ROCHA ${ }^{1}$, R.L. FIALHO ${ }^{1}$, J.J. MARQUES ${ }^{2}$, C. DARIVA ${ }^{3}$, E. FRANCESCHI ${ }^{3}$, R.B.M. \\ NUNES $^{3}$, M.A. DAMASCENA ${ }^{3}$, e F.L.P. PESSOA ${ }^{4}$ \\ ${ }^{1}$ Universidade Federal da Bahia, Programa de Pós-Graduação em Engenharia Industrial \\ ${ }^{2}$ Universidade Federal de Sergipe, Núcleo de Engenharia Ambiental \\ ${ }^{3}$ Universidade Tiradentes, Núcleo de Estudos em Sistemas Coloidais \\ ${ }^{4}$ Universidade Federal do Rio de Janeiro, Escola de Química \\ E-mail para contato: inaura.rocha@gmail.com
}

\begin{abstract}
RESUMO - O equilíbrio de fases de sistemas representativos de petróleo constitui a base para o desenvolvimento de processos dessa indústria, propiciando a concepção de equipamentos, à elaboração de previsões de produção e o aprimoramento dos métodos de recuperação. Um modo de obter conhecimento sobre o comportamento desse fluido em condições específicas de pressão, temperatura e composição é através do estudo de transição de fases. Nesse contexto, o objetivo deste trabalho consiste na obtenção de pressões de transição de fases de sistemas representativos de petróleo, constituído por um hidrocarboneto leve $\left(\mathrm{C}_{8} \mathrm{H}_{18}\right)$ e uma fração pesada na presença de $\mathrm{CO}_{2}$, via método sintético visual. Entretanto, como tais sistemas apresentam-se opacos à luz visível o uso de infravermelho se fez necessário para visualização do interior da célula. Foram verificadas transições do tipo líquido-vapor (L-V) e diagramas PTx foram elaborados para confronto dos resultados.
\end{abstract}

\section{INTRODUÇÃO}

Denomina-se petróleo às misturas multicomponentes de ocorrência natural líquida, sólida ou gasosa, composta principalmente por hidrocarbonetos (50-90\%) que se acumulam em reservatórios da subsuperfície (Pedersen e Christensen, 2007). Seus constituintes formam um espectro de hidrocarbonetos quase contínuo, do mais leve, através de pesos moleculares intermediários, até moléculas muito grandes. A proporção relativa destas diferentes peças, agregada a existência de compostos inorgânicos, pode variar numa ampla gama - o que resulta em fluidos de petróleos com características muito diferentes, ainda que em uma mesma acumulação (Stenby e Yan, 2005).

Considerando que o petróleo é uma mistura multicomponente, seu estado físico depende altamente de sua composição. Ademais, sabe-se que os reservatórios de petróleo são submetidos a 


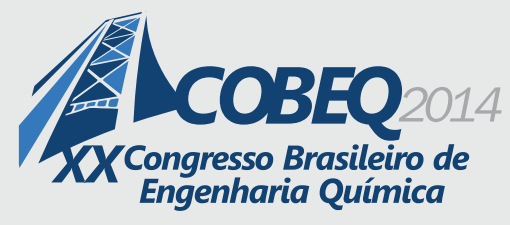

19 a 22 de outubro de 2014

Florianópolis/SC

constantes alterações de pressão e temperatura em decorrência dos seus processos produtivos. Essas alterações acontecem tanto para o material que permanece no interior da jazida como para o que está sendo produzido (neste caso, viajam do reservatório por tubulações, passam por separadores na superfície para então serem transportados em dutos). Desse modo, mudanças de fases acontecem continuamente durante a produção de petróleo, sendo um aspecto de maior importância para o entendimento do comportamento dos reservatórios (Rosa et al., 2011).

Entre outros exemplos que exprimem a importância acerca do comportamento de fases dentro da indústria de petróleo estão à elaboração de previsões de produção, o projeto de diversos equipamentos de transporte e separação, e o aprimoramento dos métodos de recuperação avançados (Whitson e Brulé, 2000). Além disto, a maior parte dos reservatórios de petróleo contém certa quantidade de frações pesadas (normalmente solubilizadas em componentes mais leves e em estado supercrítico) que podem precipitar em decorrência de alterações de temperatura e pressão, causando entupimentos nos equipamentos e dutos em diferentes estágios de exploração (Alfradique, 2006).

Apesar de um fluido de reservatório poder ser constituído por milhares de compostos, seu comportamento pode ser fundamentado através da análise de componentes puros ou misturas multicomponentes simples (Danesh, 2007). Atualmente, há disponível uma variedade de técnicas experimentais para estudo de equilíbrio de fases. A decisão sobre qual técnica pode ser mais adequada para caso depende tanto das propriedades dos sistemas de interesse como das propriedades que se pretende investigar. Cada método possui suas vantagens e desvantagens e nenhum pode ser absolutamente estabelecido como técnica universal (Robinson, 1993). A modelagem de reservatórios inclusive necessita frequentemente de informações experimentais para simular o comportamento de fases dos fluidos de reservatório (Ayala, 2007).

Sobre as técnicas experimentais para estudo de equilíbrio de fases em sistemas compostos por petróleo (opacos), normalmente faz-se o uso de métodos analíticos, e em maioria são determinadas propriedades físicas como densidade, viscosidade e solubilidade para cálculo do equilíbrio. No método sintético visual as transições de fases são detectadas por observação visual, enquanto a temperatura ou pressão são variadas, sendo a identificação das fases dependente da habilidade do observador. São sinais de transições de fases, por exemplo, o aparecimento/desaparecimento de bolhas, de menisco ou névoas em sistemas inicialmente monofásicos. Entretanto, apresenta-se inviável em meios isotrópicos (com propriedades físicas comuns entre si) nos quais as fases coexistentes têm aproximadamente o mesmo índice de refracção. Ademais, foi aplicado com sucesso em meios transparentes desde a determinação de ELV a estudos mais complexos, como no equilíbrio multifásico (Dohrn et al., 2012; Fonseca et al., 2011).

Contudo, não foram encontrados estudos envolvendo a determinação direta de pressões de transições de fases em sistemas compostos por frações pesadas de petróleo através do método sintético visual. Isto porque a aplicação desta metodologia, em seu modo tradicional, não possibilita a visualização das mudanças de fases em sistemas escuros. Diante do exposto, o presente trabalho tem por objetivo a determinação de dados de equilíbrio de fases de sistemas representativos de petróleo, formado por octano $\left(\mathrm{C}_{8} \mathrm{H}_{18}\right)$ e uma fração de petróleo pesada na presença de $\mathrm{CO}_{2}$, empregando o método sintético visual associado à luz infravermelha. 


\section{MATERIAIS E MÉTODOS}

A determinação das pressões de transição de fases do sistema composto por $\mathrm{CO}_{2}$, octano e fração pesada $(\mathrm{F} 4)$ foi realizada em uma unidade de alta pressão conforme o método sintético visual, o que significa que nenhuma amostragem é necessária. A proporção da $\mathrm{F} 4$ de petróleo foi mantida em 1 e $10 \%(\mathrm{~m} / \mathrm{m}) \mathrm{C}_{8} \mathrm{H}_{18}: \mathrm{FP}$, e as temperaturas de interesse foram $\mathrm{T}=30,50$ e $70^{\circ} \mathrm{C}$.

O teor de F4 foi variado com o propósito de avaliar a sua influência no comportamento de fases e o potencial do novo esquema experimental, projetado para esses tipos de sistemas. As misturas líquidas eram preparadas separadamente, de modo que cada amostra "de óleo" a ser estudada contivesse o percentual pré-estabelecido de F4.

O octano é proveniente da Sigma-Aldrich, com 99\% de pureza; o $\mathrm{CO}_{2}$ da White Martins, com 99,9\%; e a F4, oriunda da destilação de um "óleo morto", constitui o resíduo de vácuo do processo. A F4 é de textura espessa e cor preta. Sua caracterização quanto ao: i) teor de água $(0.087 \%), 2)$ densidade $\left(\rho=8.970 \mathrm{~g} / \mathrm{cm}^{3}\right.$ a $50^{\circ} \mathrm{C}$ ) e 3) pressão de vapor (equivalente ao $\mathrm{C}_{28} \mathrm{H}_{58}$ ) está discutida minuciosamente no trabalho de Lucas (2013).

\subsection{Aparato Experimental}

Os experimentos foram conduzidos em uma unidade de alta pressão, conforme mostra a Figura 1, seguindo a metodologia sintética visual, através de uma célula de volume variável para observação das transições de fases dos sistemas de petróleo.

A célula é feita em aço inoxidável, com um volume interno máximo de $8 \mathrm{~cm}^{3}$ e é composta por: corpo cilíndrico; pistão, conexões para termopar e injeção de solvente, uma janela frontal e duas janelas laterais (equipadas com safiras). Buscando a visualização do interior da célula foram empregados uma fonte de luz infravermelha (780-1400 nm, Philips) e um microscópio com capacidade de ampliação 200x (Dino-Lite Digital Microscope, modelo AM413FIT). 


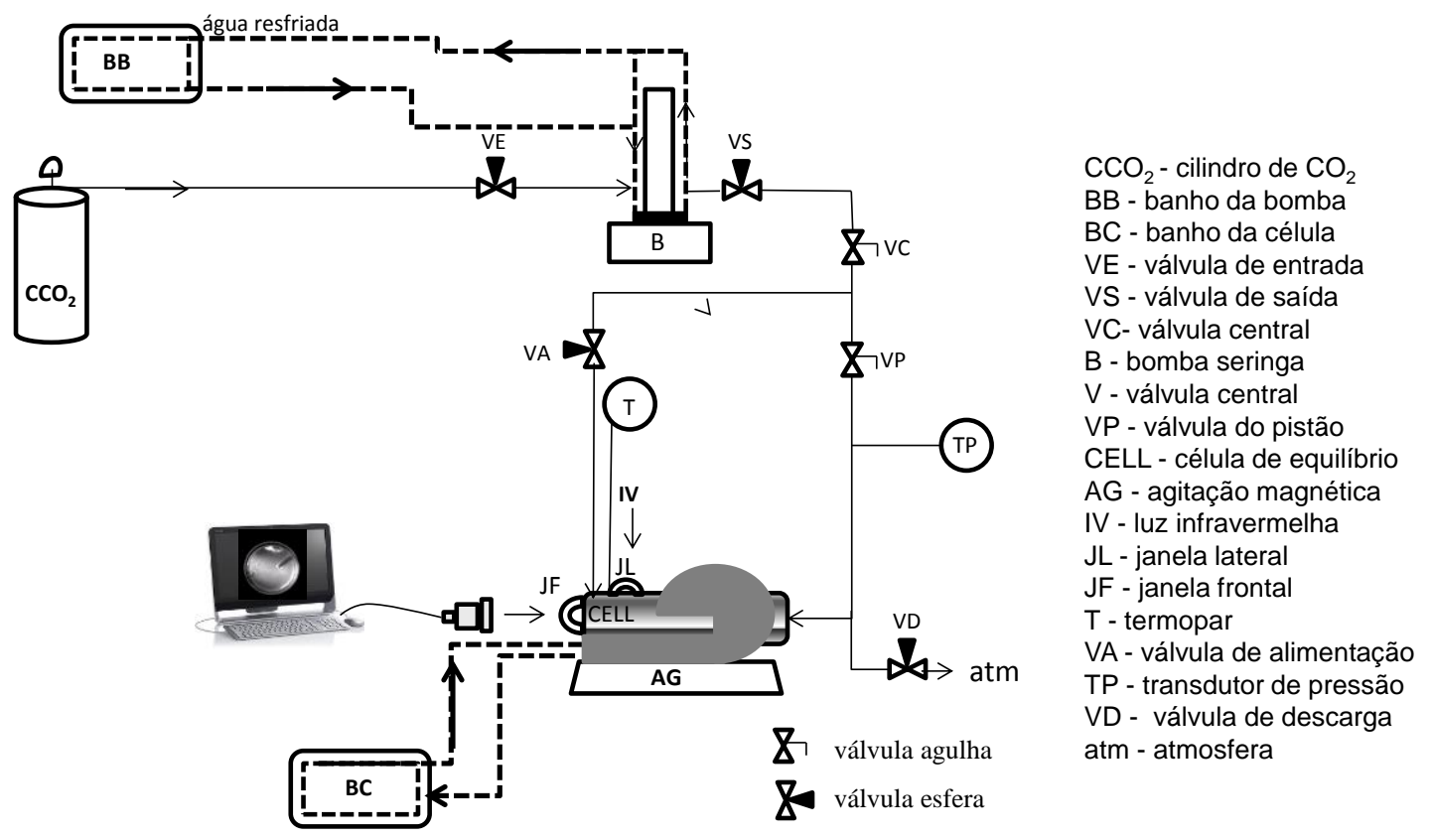

Figura 1 - Aparato experimental

Procedimento Experimental: Basicamente, o procedimento de obtenção dos dados consiste na pressurização de uma mistura (empregando o próprio $\mathrm{CO}_{2}$ como fluido pressurizador) a uma dada temperatura, com incremento de pressão até que o mesmo encontre-se em uma única fase. A partir desse momento, é feita uma lenta despressurização do sistema até que o mesmo volte a

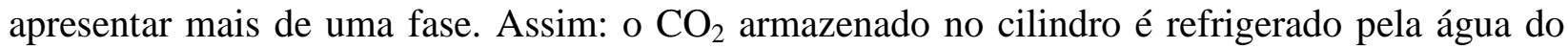
banho e enviado para o reservatório da bomba de seringa. A água resfriada circula entre a jaqueta da bomba de seringa e o banho de gelo. Para a aquisição de dados de pressão, um indicador de pressão deve estar ligado a um transdutor estrategicamente posicionado próximo a válvula que permite a pressurização da célula pelo pistão. O controle de temperatura é realizado por uma camisa de alumínio, envolta da célula, por onde circula água aquecida por um banho térmico. A tomada de temperatura é realizada no seio da mistura através da introdução de um termopar tipo J em um dos orifícios da célula de equilíbrio. Um agitador magnético, controlado por uma placa de agitação magnética, é inserido no interior da célula para auxiliar o alcance do estado de equilíbrio da mistura. A mistura é comprimida até a pressão desejada, devido o fluido pressurizador movimentar o pistão localizado no interior da célula. Um tempo é requerido para estabilização da mistura na temperatura de interesse. Para visualizar o interior da célula, é posicionada na janela lateral uma luz IV e na janela frontal o microscópio conectado a um computador. A mistura é lentamente despressurizada e após a determinação da mudança de fases em uma isoterma, a célula é aquecida até uma nova temperatura, pressurizada até visualização de uma única fase, para então serem medidas novas pressões de transição. 


\section{RESULTADOS E DISCUSSÃO}

As pressões de transição de fases para o sistema $\left(\mathrm{CO}_{2} / \mathrm{C}_{8} \mathrm{H}_{18} / \mathrm{F} 4\right)$ a $\mathrm{T}=30,50$ e $70{ }^{\circ} \mathrm{C}$ estão listadas na Tabela 1. A tabela exibe para cada teor de F4 na mistura e por composições em massa de $\mathrm{CO}_{2}\left(\mathrm{x}_{1}\right)$, os valores de pressão medidos (bar), os desvios padrões absolutos $(\sigma)$ e a natureza da transição de fases. Gráficos p versus $\mathrm{x}_{1}$ foram elaborados para representar as pressões de transição de fases medidas que variaram entre 27,9 e 195 bar. Para a faixa de estudo empregada foi observado equilíbrio do tipo L-V. Portanto, os sistemas foram avaliados conforme: i) às diferentes influências de F4 na mistura; ii) à variação da composição global, e iii) à variação de temperatura $\left(\mathrm{T}=30,50\right.$ e $\left.70^{\circ} \mathrm{C}\right)$.

Tabela 1 - Pressões de transição para o sistema $\left(\mathrm{CO}_{2(1)} / \mathrm{C}_{8} \mathrm{H}_{18} / \mathrm{F} 4\right)$.

\begin{tabular}{|c|c|c|c|c|c|c|c|c|c|c|}
\hline & \multirow{2}{*}{$x_{1}$} & \multicolumn{3}{|c|}{$\mathrm{T}\left(30^{\circ} \mathrm{C}\right)$} & \multicolumn{3}{|c|}{$\mathrm{T}\left(50^{\circ} \mathrm{C}\right)$} & \multicolumn{3}{|c|}{$\mathrm{T}\left(70^{\circ} \mathrm{C}\right)$} \\
\hline & & P (bar) & $\sigma$ (bar) & Tipo & P (bar) & $\sigma($ bar $)$ & Tipo & P (bar) & $\sigma$ (bar) & Tipo \\
\hline \multirow{5}{*}{$\begin{array}{c}1 \% \\
(\mathrm{~m} / \mathrm{m}) \\
\mathrm{F} 4: \mathrm{C}_{8} \mathrm{H}_{18}\end{array}$} & 0,25 & 27,9 & 2,40 & $\mathrm{~PB}$ & 44,8 & 0,21 & $\mathrm{~PB}$ & 59,8 & 0,07 & PB \\
\hline & 0,50 & 37,8 & 0,42 & PB & 66,5 & 0,64 & PB & 85,5 & 2,83 & PB \\
\hline & 0,75 & 48,1 & 0,78 & PB & 74,1 & 0,42 & PB & 105,0 & 0,07 & PB \\
\hline & 0,85 & 59,0 & 0,00 & PB & 94,0 & 0,00 & PO & 124,5 & 0,71 & PO \\
\hline & 0,95 & 61,2 & 1,77 & PB & 86,2 & 0,21 & PO & 110,1 & 0,35 & PO \\
\hline \multirow{5}{*}{$\begin{array}{c}10 \% \\
(\mathrm{~m} / \mathrm{m}) \\
\mathrm{F} 4: \mathrm{C}_{8} \mathrm{H}_{18}\end{array}$} & 0,25 & 38,6 & 0,21 & PB & 63,2 & 0,92 & PB & 79,5 & 0,71 & PB \\
\hline & 0,50 & 44,7 & 0,64 & PB & 90,6 & 4,24 & PB & 117,8 & 4,88 & PB \\
\hline & 0,75 & 97,0 & 3,68 & PB & 143,6 & 3,96 & PB & 195,0 & 7,07 & PB \\
\hline & 0,85 & 90,3 & 0,42 & PB & 132,5 & 3,46 & $\mathrm{PO}$ & 162,7 & 0,21 & $\mathrm{PO}$ \\
\hline & 0,95 & 82,0 & 1,48 & PB & 118,2 & 0,14 & PO & 143,3 & 4,60 & $\mathrm{PO}$ \\
\hline
\end{tabular}

A utilização de luz infravermelha foi crucial para visualização das mudanças de fases. Pressões da bolha, apresentadas na Figura $2-2$, foram observadas com distinção até $\mathrm{x}_{1}=0.5$, enquanto que em composições de $75 \% \mathrm{CO}_{2}$ o aparecimento de bolhas foi diferenciado, levando mais tempo para se definir. Este comportamento pode ser explicado pela proximidade do ponto crítico. Pontos de orvalho geraram condensado, como exibe a Figura 3 , e foram sempre observados para $\mathrm{x}_{1}=0.85$ e 0.95 acima de $\mathrm{T}=50^{\circ} \mathrm{C}$. 


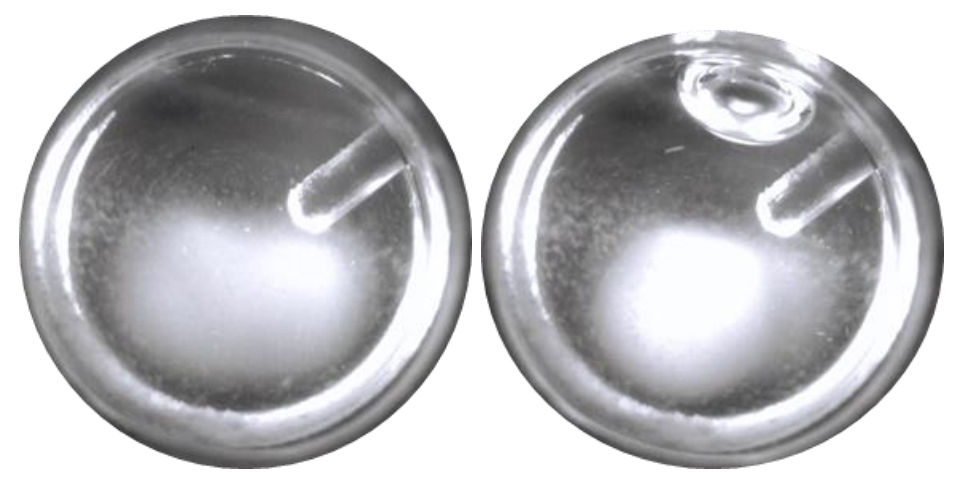

Figura 2 - Pressão tipo bolha para os sistemas $\left(\mathrm{CO}_{2} / \mathrm{C}_{8} \mathrm{H}_{18} / \mathrm{F} 4\right)$ com $50 \% \mathrm{CO}_{2}$ e $1 \%(\mathrm{~m} / \mathrm{m}) \mathrm{F} 4: \mathrm{C}_{8} \mathrm{H}_{18}$

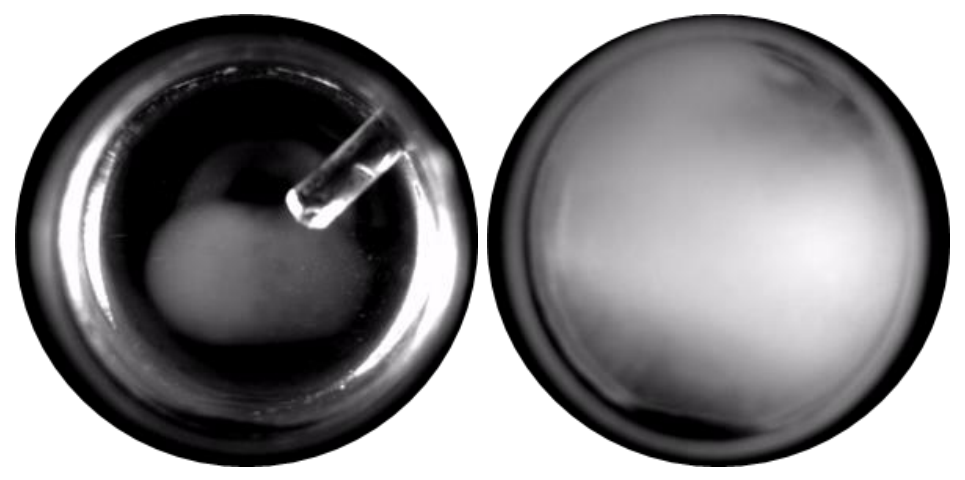

Figura 3 - Pressão tipo orvalho para os sistemas $\left(\mathrm{CO}_{2} / \mathrm{C}_{8} \mathrm{H}_{18} / \mathrm{F} 4\right)$ com $95 \% \mathrm{CO}_{2}$ e $1 \%(\mathrm{~m} / \mathrm{m}) \mathrm{F} 4: \mathrm{C}_{8} \mathrm{H}_{18}$

Observando a Figura 4, percebe-se que as pressões experimentais do sistema $\left(\mathrm{CO}_{2} / \mathrm{C}_{8} \mathrm{H}_{18} / \mathrm{F} 4\right)$ aumentam conforme a temperatura é elevada, confirmando a dependência desta propriedade com a temperatura. A influência da fração F4 sobre as pressões de transição de fases também é verificada ao examinar a Figura 4 (a), (b) e (c), na qual as pressões para cada teor de $\mathrm{F} 4$ foram agrupadas por temperatura. Ademais, dados de ELV a $49.24^{\circ} \mathrm{C}$ para o sistema $\left(\mathrm{CO}_{2} / \mathrm{C}_{8} \mathrm{H}_{18}\right)$, por Jimenéz-Gallegos et al. (2006), foram acrescentados ao gráfico para análise do efeito da F4. Notou-se que: (i) as curvas sofrem influência da presença da $\mathrm{F} 4$ na região rica em $\mathrm{CO}_{2}$; ii) as pressões experimentais são maiores quando se aumenta a quantidade de F4; (iii) a F4 desloca o ponto crítico da mistura para a esquerda, antecipando este ponto à medida que o seu teor é maior. 

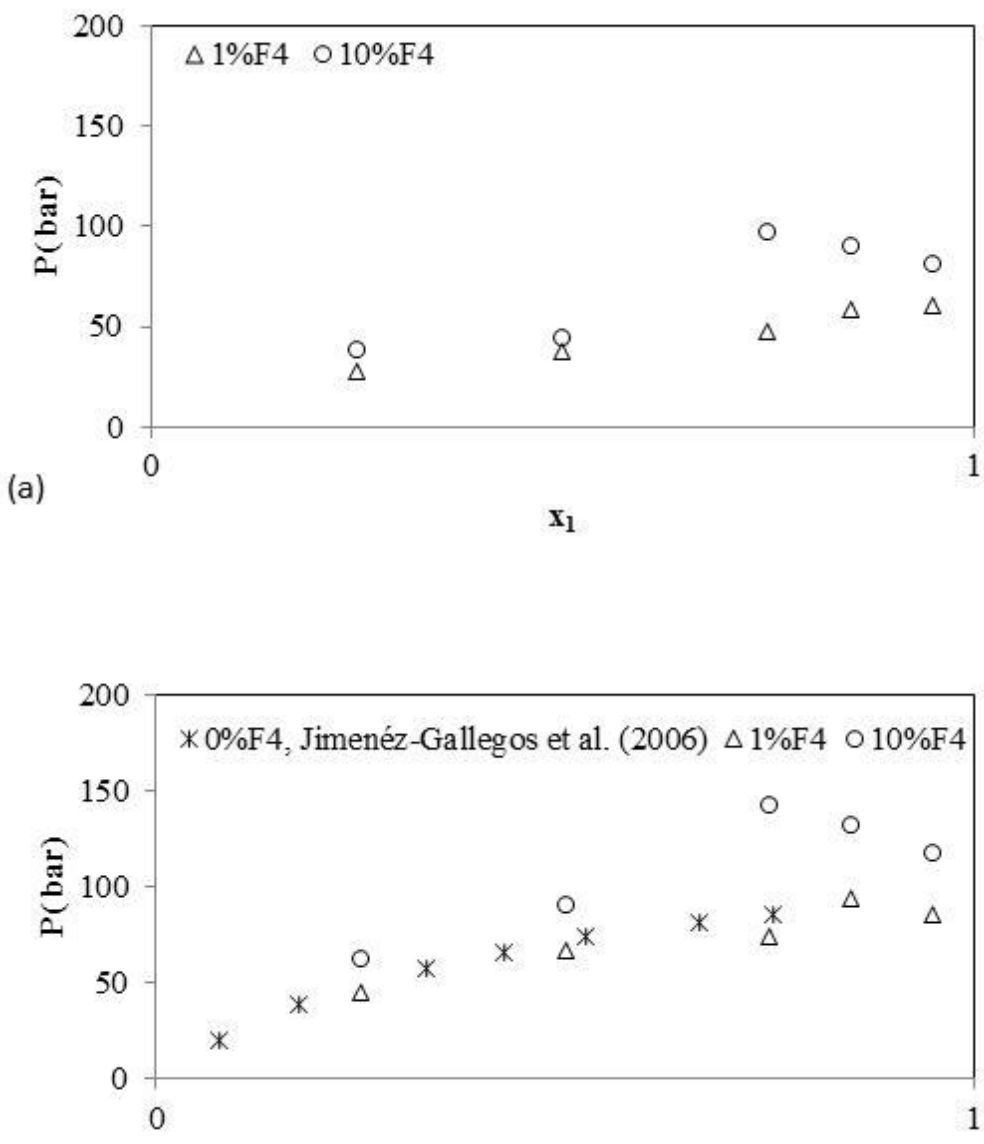

(b)

$\mathrm{x}_{1}$

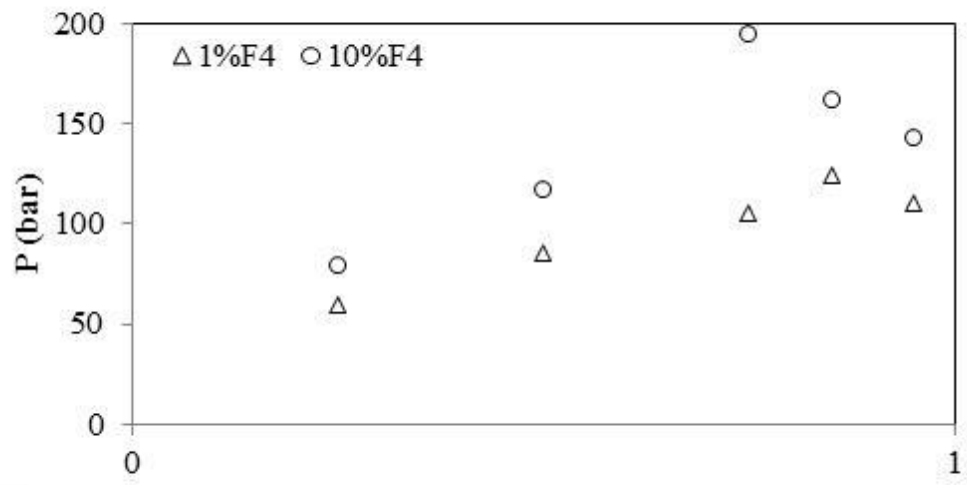

(c)

$\mathrm{x}_{1}$

Figura 4 - Influência da F4 no sistema $\left(\mathrm{CO}_{2} / \mathrm{C}_{8} \mathrm{H}_{18} / \mathrm{F} 4\right)$. (a) $30^{\circ} \mathrm{C}$ (b) $50^{\circ} \mathrm{C}$; (c) $70^{\circ} \mathrm{C}$. 


\section{REFERÊNCIAS}

ALFRADIQUE, M. F. Cálculo de equilíbrio de fases em alta pressão aplicado à indústria do petróleo. Tese (Doutorado em Engenharia Química). Universidade Federal do Rio de Janeiro, Rio de Janeiro, 2006.

AYALA, L. F. Phase behaviour of hydrocarbon fluids: the key to understanding oil and gas engineering systems. Business Briefing: Oil \& Gas Processing Review, 2006.

DANESH, A. PVT and phase behaviour of petroleum reservoir fluids. Amsterdam: Editora Elsevier, 2007.

DOHRN, R.; FONSECA, J. M.S.; PEPER, S. Experimental methods for phase equilibria at high pressures. Annu. Rev. Chem. Biomol. Eng., v. 3, p. 343-67, 2012.

FONSECA, J.M.S.; DOHRN, R.; PEPER, S. High-pressure fluid phase equilibria: experimental methods and systems investigated (2005-2008). Fluid Phase Equilib., v. 300, p. 1-69, 2011.

JIMÉNEZ-GALLEGOS, R.; GALICIA-LUNA, L.A.; ELIZALDE-SOLIS, O. Experimental vaporliquid equilibria for the carbon dioxide + octane and carbon dioxide + decane systems. J. Chem. Eng. Data, v. 51, p. 1624-1628, 2006.

LUCAS, M. A. Desenvolvimento de Ferramentas para estudo do Comportamento de Fases em Sistemas a Alta Pressão contendo Petróleo e $\mathrm{CO}_{2}$ : fracionamento e espectroscopia NIR. 97p. Dissertação (Mestrado em Engenharia de Processos), Universidade Tiradentes, Aracaju, 2013.

PEDERSEN, K. S.; CHRISTENSEN, P. L. Phase behavior of petroleum reservoir fluids. Nova York: CRC/Taylor \& Francis, 2007.

ROBINSON, B.D. Experimental methods for measurement of phase equilibria at high pressures. Pure \& Appl. Chem., v.65, No. 5, 971-976, 1993.

ROSA, A. J.; CARVALHO, R. S.; XAVIER, J. A. D. Engenharia de reservatórios de petróleo. $1^{\text {a }}$. ed. Rio de Janeiro: Editora Interciência, 2011.

STENBY, E. A.; YAN, W. Encyclopedia of hydrocarbons: exploration, production and transport. Itália: Editora Istituto Della Enciclopedia Italiana Fondata da Giovanni Treccani S.P.A., 2005.

WhitSON, C. H.; BRULÉ, M. R. Phase Behavior-SPE Monograph Henry L. Doherty Series. Richardson: SPE, v. 20, 2000. 